\title{
NEW TAXA AND COMBINATIONS IN THE GENUS BROSIMUM (MORACEAE)
}

\author{
C. C. BERG
}

Instituut voor Systematische Plantkunde, Utrecht

The sequence in which the nomenclatural and taxonomic novelties in the genus Brosimum appear below corresponds with that in the revision of the neotropical Brosimeae to be published in the near future.

Brosimum alicastrum Swartzs sp. bolivarense (Pittier) C. C. Berg nov. comb. et stat.

Basionym: Helicostylis bolivarensis Pittier, Contr. U. S. Nat. Herb. 20: 96. 1918.

Brosimum acutifolium Huber ssp. interjectum C. C. Berg nov. subsp.

Folia oblonga vel elliptica vel (sub)ovata, 6-16 cm longa, 4-6 cm lata, acuminata, basi obtusa, emarginata vel subcordata, supra vix scabridula, subtus puberula; venulae paulo prominulae; petioli 4-6 $\mathrm{mm}$ longi.

Type: Ducke s.n. (HJBR 19477), Brazil, Pará, Juruty Velha, near the western boundary of Pará $(\mathrm{U})$.

Brosimum acutifolium Huber ssp. obovatum (Ducke) C. C. Berg nov. comb. et stat.

Basionym: Brosimopsis obovata Ducke, Bull. Mus. Hist. Nat. Paris II. 4: 722. 1932.

Brosimum Swartz sect. Brosimopsis (S. Moore) C. C. Berg nov. stat.

Basionym: Brosimopsis S. Moore, Trans. Linn. Soc. II. 4: 473. 1895.

Brosimum lactescens (S. Moore) C. C. Berg nov. comb.

Basionym: Brosimopsis lactescens S. Moore, Trans. Linn. Soc. II. 4: 473. 1895.

Brosimum Swartz sect. Piratinera (Aublet) C. C. Berg nov. stat.

Basionym: Piratinera Aublet, Pl. Gui. 2: 888. 1775. 
NEW TAXA AND COMBINATIONS IN THE GENUS BROSIMUM

Brosimum Swartz subg. Ferolia (Aublet) C. C. Berg nov. stat.

Basionym: Ferolia Aublet, Pl. Gui. Suppl. 7. 1775.

Brosimum melanopotamicum C. C. Berg nov. spec.

Arbor dioica. Folia elliptica vel oblonga, $5.5-11.5 \mathrm{~cm}$ longa, $3.5-6 \mathrm{~cm}$ lata, subtus puberula vel hirtella, ad costam venas secundariasque appresse pubescentia; venatio grossa; venae supra impressae vel planae, subtus prominentes; venae secundariae arcuantes procul a margine; stipulae 3-7 $\mathrm{mm}$ longae. Inflorescentiae masculae multiflorae; perianthium $0.3-0.5 \mathrm{~mm}$ altum, tepalis 2-4 inaequabilibus; stamina 1-4, saepe 2 .

Type: Pires 247, Brazil, Amazonas, Santa Isabel, on Rio Negro (US).

Brosimum utile (H.B.K.) Pittier ssp. occidentale C. C. Berg nov. subsp.

Folia elliptica vel oblonga, medio latitudine maxima, $7.5-13.5 \mathrm{~cm}$ longa, 4.5-7 cm lata, basi acuta vel obtusa; margine integro; venae secundariae utrinque 18-21, supra impressae; stipulae $2.5-5.5 \mathrm{~cm}$ longae. Inflorescentiae solitariae, pedunculo 3-4 mm longo; receptaculum non vel vix foveatum; flores masculi multi; perianthium $0.2-0.8 \mathrm{~mm}$ altum; stamen unicum; filamenta 1-1.5 mm longa, antherae $0.4-0.5 \mathrm{~mm}$ longae, $0.5-0.6 \mathrm{~mm}$ latae; stigmata circ. $2 \mathrm{~mm}$ longa.

Type: Cuatrecasas 16084, Colombia, Dept. del Valle, between El Aguacante and Quebrada de la Yuca (F).

Brosimum utile (H.B.K.) Pittier ssp. allenii (Woodson) C. C. Berg nov. stat.

Basionym: Brosimum allenii Woodson, Ann. Missouri Bot. Gard. 47: 128. 1960.

Brosimum utile (H.B.K.) Pittier ssp. magdalenense C. C. Berg nov. subsp.

Folia elliptica vel oblonga, latitudine maxima sub medio, 9.5-17 cm longa, 6-8.5 cm lata, basi emarginata vel subcordata; margine integro; venae secundariae utrinque 18-23, supra fere planae; stipulae circ. $1.5 \mathrm{~cm}$ longae. Inflorescentiae solitariae, pedunculo $3-5 \mathrm{~mm}$ longo; receptaculum lob(ul)atum et foveolatum; flores masculi multi; perianthium $0.1-0.2 \mathrm{~mm}$ altum; stamen unicum; filamenta $0.5-0.6 \mathrm{~mm}$ longa, antherae circ. $0.3 \mathrm{~mm}$ longae, circ. $0.2 \mathrm{~mm}$ latae; stigmata $0.6-0.8 \mathrm{~mm}$ longa.

Type: Lawrance 765, Colombia, Boyaca, El Humbo (US).

Brosimum utile (H.B.K.) Pittier ssp. darienense C. C. Berg nov. subsp.

Folia oblonga vel lanceolata, latitudine maxima prope medium, $12-20 \mathrm{~cm}$ longa, 5.5-8 cm lata, basi obtusa; margine integro; venae secundariae utrinque 
C. C. BERG

23-29, supra fere planae; stipulae $1.5-3 \mathrm{~cm}$ longae. Inflorescentiae solitariae, pedunculo 10-12 $\mathrm{mm}$ longo; receptaculum foveatum; flores masculi multi; perianthium 0.1-0.3 mm altum; stamen unicum; filamenta $0.5-0.7 \mathrm{~mm}$ longa, antherae circ. $0.3 \mathrm{~mm}$ longae, circ. $0.2 \mathrm{~mm}$ latae; stigmata $0.2-0.8 \mathrm{~mm}$ longa.

Type: Pittier 4418, Panama, San Blas Coast, hills of Sperdi, near Puerto Obaldía (US).

Brosimum utile (H.B.K.) Pittier ssp. longifolium (Ducke) C. C. Berg nov. stat. Basionym: Brosimum longifolium Ducke, Arq. Serv. Flor. Rio de Janeiro 1 : 3. 1 y3y.

Brosimum utile (H.B.K.) Pittier ssp. ovatifolium (Ducke) C. C. Berg nov. stat. Basionym: Brosimum ovatifolium Ducke, Arch, Jard. Bot. Rio de Janeiro 3: 25.1922.

Brosimum parinarioides Ducke ssp. amplicoma (Ducke) C. C. Berg nov. stat. Basionym: Brosimum amplicoma Ducke, Tropical Woods 31: 10.1932.

\section{ACKNOWLEDGEMENT}

The author is much indebted to Dr. K. U. Kramer who contributed the latin diagnoses. 